\title{
Fuegos cruzados. Bourdieu, la crítica a la fenomenología social y el habitus*
}

\author{
[Versión en castellano] \\ Crossfires. Bourdieu, Criticism of Social Phenomenology and Habitus
}

Fogos cruzados. Bourdieu, critica à fenomenología e ao habitus social Recibido el 11 de febrero, 2020. Aceptado el 13 de abril, 2020.

Juan Dukuen**
https://orcid.org/0000-0001-5089-1858

Argentina

Para citar este artículo:

Dukuen, Juan (2020). Fuegos

Cruzados. Bourdieu, la crítica a la fenomenología social y el habitus.

Ánfora, 27(49), 17-42.

https://doi.org/10.30854/anfiv27.n49.2020.734

Universidad Autónoma de

Manizales. ISSN 0121-6538/

e-ISSN 2248-6941.

CC BY-NC-SA 4.0

\section{Resumen}

Objetivo: proponer una lectura alternativa de la noción de habitus de Bourdieu inspirada en la fenomenología de Merleau-Ponty, con base en el análisis de la crítica bourdeana a la "fenomenología social" y de las objeciones producidas por sociólogos de orientación fenomenológica. Metodología: se aplicó una lectura analítica de las obras de Bourdieu, Merleau-Ponty y de las exégesis críticas de orientación fenomenológicas de Thoop \& Murphy y Belvedere. Resultados: se objeta en Throop \& Murphy la acusación sobre el determinismo del habitus y la falta de aportes de Bourdieu, señalando el desarrollo de una teoría de la dominación merleaupontyana centrada en la formación de habitus corporales. Frente a las críticas de Belvedere a la noción de habitus, se

\footnotetext{
* Este artículo presenta resultados parciales de una investigación realizada entre 2008 y 2019. Cuenta con el aval del PICT 2017-0661 (ANPCyT Argentina) Dir: Dra. M. Kriger y del PUE 22920160100005 CO-CIS CONICET/IDES-Argentina. Dir: Dr. S. Visacovsky. Una primera versión fue presentada en el 1er Congreso Latinoamericano de Teoría Social-UBA, 2015. El autor declara que no hubo conflicto de intereses en la ejecución del proyecto de investigación.

** Doctor en Ciencias Sociales. Investigador Adjunto del Consejo Nacional de Investigaciones Científicas y Técnicas (Argentina). Profesor de la Universidad de Buenos Aires, Argentina. Correo electrónico: juandukuen@gmail.com
} 
respondió con una reelaboración basada en Merleau-Ponty donde el cuerpo-agente es parcialmente estructurado por un habitus entrelazado a una dimensión actual. Contra el teoricismo y el determinismo, se ilustró con resultados de una investigación sobre becarias de un colegio de clases altas de Buenos Aires, Argentina. Conclusiones: se concluye que las críticas a Bourdieu caen en el teoricismo, obviando los análisis empíricos donde se apropia de la fenomenología. Se señala que recuperando la perspectiva merleaupontyana se puede fundamentar una antropología reflexiva de los poderes y modos de dominación y restituir la praxeología a la tradición fenomenológica.

Palabras-clave: Bourdieu; Fenomenología social; Sociología; Etnometodología; Schutz; Habitus; Merleau-Ponty.

\section{Abstract}

Objective: to propose an alternative reading of Bourdieu's notion of habitus inspired by the phenomenology of Merleau-Ponty, based on the analysis of Bourdieu's criticism of "social phenomenology" and of the objections produced by sociologists with a phenomenological orientation. Methodology: an analytical reading of the works of Bourdieu, Merleau-Ponty and of the phenomenologically oriented critical exegeses of Thoop \& Murphy and Belvedere was applied. Results: Throop and Murphy object to the accusation about the determinism of habitus and Bourdieu's lack of input, pointing to the development of a Merleau-Pontyan theory of domination focused on the formation of corporal habitus. Faced with Belvedere's criticism of the notion of habitus, a reply was proposed, consisting on a reworking based on Merleau-Ponty in which the agent-body is partially structured by a habitus intertwined to a actual dimension. In opposition to theoricism and determinism, the proposal was illustrated with the results of an investigation of scholarship students from upper class high school in Buenos Aires, Argentina. Conclusions: it is concluded that the criticisms of Bourdieu fall into theoricism, ignoring the empirical analyses where phenomenology is appropriated. In addition, it is pointed out that by recovering the Merleaupontian perspective, a reflexive anthropology of the powers and modes of domination can be founded and praxeology can be restored to the phenomenological tradition.

Keywords: Bourdieu; Social phenomenology; Sociology; Ethnomethodology; Schutz; Habitus; Merleau-Ponty. 


\section{Resumo}

Objetivo: propor uma leitura alternativa da noção de habitus de Bourdieu inspirada na fenomenologia de Merleau-Ponty, com base na análise da crítica de Bourdieu à "fenomenologia social" e das objeções produzidas por sociólogos com orientação fenomenológica. Metodologia: foi aplicada uma leitura analítica dos trabalhos de Bourdieu, Merleau-Ponty e da exegese crítica orientada à fenomenologia de Thoop \& Murphy e Belvedere. Resultados: A acusação sobre o determinismo do habitus e a falta de contribuições de Bourdieu, objetivando o desenvolvimento de uma teoria de dominação Merleaupontyana focada na formação do habitus corporais, é objetada por Throop \& Murphy. Diante da crítica de Belvedere à noção de habitus, foi respondida com um retrabalho baseado em Merleau-Ponty, onde o corpo do agente é parcialmente estruturado por um habitus entrelaçado a uma dimensão atual. Contra o teorismo e o determinismo, foi ilustrado com os resultados de uma investigação de bolsistas de uma faculdade de alta classe em Buenos Aires, Argentina. Conclusões: conclui-se que as críticas de Bourdieu se enquadram no teorismo, ignorando as análises empíricas em que a fenomenologiaé apropriada. conclui-se que as críticas de Bourdieu se enquadram no teorismo, ignorando as análises empíricas em que a fenomenologia é apropriada. Assinala-se que, ao recuperar a perspectiva merleaupontiana, uma antropologia reflexiva dos poderes e modos de dominação pode ser fundada e a praxeologia pode ser restaurada à tradição fenomenológica.

Palavras-chave: Bourdieu; Fenomenologia social; Sociologia; Etnometodologia; Schutz; Habitus; Merleau-Ponty. 


\section{Introducción}

En las últimas tres décadas diversos estudios señalan la relación productiva entre la teoría de la práctica de Pierre Bourdieu y la tradición fenomenológica (Heran, 1987; Hong, 1999; Throop y Murphy, 2002; Haber, 2004, Martin-Criado, 2006; Bimbenet, 2011; Martínez, 2007; Ralón, 2010; Ralón y Dukuen, 2013; Csordas, 2011; Dukuen, 2010, 2011, 2013, 2015, 2016, 2018b; Perreau, 2019).

Análisis recientes muestran que ya en sus primeras investigaciones empíricas sobre la imposición brutal del capitalismo en la sociedad argelina y el celibato de los primogénitos en el Béarn francés, Bourdieu (1962, 1963), filósofo devenido etnólogo, realiza una "antropologización" de problemáticas fenomenológicas (Martínez, 2007; Dukuen, 2011, 2013, 2018b) referidas al cuerpo propio (Leib) y la temporalidad pre-objetiva que encuentran fundamento en las investigaciones de Husserl (2002) y Merleau-Ponty (1942, 1945). Esta "antropologización” contribuirá a la génesis del concepto bourdeano de habitus como "sistema de disposiciones" ${ }^{1}$ (que convive inicialmente con ethos y hexis, en la extensa genealogía que va de Aristóteles (1997) a Tomás de Aquino (1993), reactivada en el siglo XX por Husserl (1966), Merleau-Ponty (1945), Durkheim (1990), Weber (2009) y Mauss (1979)) y a la formación de la teoría disposicional de la práctica o praxeología entre las décadas de 1960 y 1970 (Hong, 1999; Martínez, 2007).

Sin embargo, cabe recordar que en la primera sistematización de la praxeología, en Esquisse d'une théorie de la pratique, Bourdieu (1972) ha mostrado una actitud crítica con el modo de conocimiento fenomenológico, que categorizará a partir de Le sens pratique (Bourdieu, 1980a) como un "subjetivismo" expresado por Sartre en filosofía, y por Schutz (1993) y Garfinkel (1967) en sociología. La lectura fallida que realiza sobre todo de Schutz hace que no sea tarea fácil comprender la praxeología como una antropología de orientación fenomenológica (Dukuen, 2013, 2018b) porque ha generado un rechazo -bien fundamentado- de quienes retoman la tradición fenomenológica en ciencias sociales (Throop y Murphy, 2002; Belvedere, 2011a, 2011b, 2012, 2013; Endress, 2005; Dreher, 2014, $2019^{2}$ ).

Teniendo en cuenta que la inspiración de la obra de Bourdieu en la tradición fenomenológica es cuestionada con centro en la noción de habitus, en este escrito se describe la crítica de Bourdieu a la "fenomenología social", luego se retoman

\footnotetext{
1. La definición estándar de habitus se encuentra en Le sens pratique: "Los condicionamientos asociados a una clase particular de condiciones de existencia producen habitus, sistemas de disposiciones duraderas y transferibles, estructuras estructuradas predispuestas a funcionar como estructuras estructurantes, es decir, como principios generadores y organizadores de prácticas y de representaciones" (Bourdieu, 1980a, pp.88-89).
}

2. Para una crítica de la crítica desarrollada por Dreher (2014) en torno a una fenomenología del poder, consultar a Dukuen (2018b). 
las objeciones agudas realizadas por Belvedere y Throop y Murphy para, a partir de ello, proponer una lectura alternativa inspirada en la fenomenología de Merleau-Ponty.

\section{Metodología}

Este trabajo se basa en una lectura analítica de las obras de Bourdieu ${ }^{3}$, a partir de la cual se constituyó un corpus específico relativo a sus referencias al modo de conocimiento fenomenológico en ciencias sociales. Por eso se centra especialmente en Esquisse y Le sens pratique. No se tratan aquí, como sí se hace en Dukuen (2018b), las críticas a Sartre y Husserl, que refieren específicamente a diferencias y diferendos en torno a la ontología y la reducción fenomenológicas.

El artículo tampoco se detiene en los Cursos de Sociología General de Bourdieu $(2015,2016)$ porque no agregan matices pertinentes para el debate propuesto y tienen un estatuto teórico y una función pedagógica diferente a los textos de investigación/reflexivos/programáticos, que sí se analizan aquí. Atendiendo a la abultada bibliografía sobre Bourdieu (Dukuen, 2013, 2018b), este trabajo se apoya en las exégesis imprescindibles. En relación con las críticas de orientación fenomenológica, se analizan las de Throop y Murphy y Belvedere relativas al concepto de habitus, con y contra las cuales se propone una reelaboración basada en una lectura de la fenomenología de Merleau-Ponty.

\section{Resultados}

\section{La critica de Bourdieu a "la fenomenologia social"}

Para comprender las críticas de Bourdieu hacia la llamada "fenomenología social" (Bourdieu, 1980a pp. 43-45), se retoma su primer análisis de esa perspectiva -con antecedentes en Un art moyen (1965, p. 22)- realizado en Esquisse de 1972. Este texto clave (germen de Le sens pratique de 1980) comienza con un acápite titulado "El observador observado", que es una crítica al modo de conocimiento teórico objetivista, expresado para Bourdieu en la etnología estructural de Lévi-Strauss (2001) bajo el lenguaje de la regla, y que se extiende y profundiza en el segundo apartado titulado "Los tres modos de conocimiento teórico". Allí se señala que el mundo social puede ser abordado mediante esos "tres modos", que si bien se diferencian según las tesis antropológicas que presentan, tienen en

3. Todas las traducciones son del autor de este artículo. 
común el hecho de que "se oponen al modo de conocimiento práctico" (Bourdieu, 1972, p. 162). Esos modos de conocimiento teóricos son el fenomenológico, el objetivista y el praxeológico, y cada uno de ellos entraña más o menos explícitamente una teoría de la práctica diferente.

Bourdieu centra la crítica en el objetivismo, extendiéndola a la lingüística de Saussure (1980) como fundamento del estructuralismo de Lévi-Strauss (2001). Llama la atención que la crítica al punto de vista fenomenológico es menor en comparación a la realizada al "objetivismo", siendo que el análisis crítico de la obra de Sartre como fundamento de esta posición, aparece en una extensa nota al pie (Bourdieu, 1972, pp. 248-250) y no ocupa un capítulo completo -La antropología imaginaria del subjetivismo- como sí lo hará en Le sens pratique. El modo de conocimiento praxeológico, donde la noción de habitus juega un papel central, será presentado aquí como una superación de los errores en que incurrirían los otros dos modos.

Siguiendo el objetivo de este trabajo, así presenta Bourdieu la caracterización del modo de conocimiento fenomenológico:

El conocimiento que llamaremos fenomenológico (o si vamos a hablar en términos de escuelas actualmente existentes "interaccionista" o "etnometodológico") explicita la verdad de la experiencia primera del mundo social, es decir, la relación de familiaridad con el entorno familiar, aprehensión del mundo social como mundo natural y evidente, sobre la cual por definición no se reflexiona, y que excluye la pregunta por sus condiciones de posibilidad (Bourdieu, 1972, pp. 162-163).

En primer lugar, Bourdieu explicita un modo de conocimiento que busca dar cuenta de la experiencia práctica de los agentes en su relación "natural y evidente" con el mundo vivido. Consideramos que esta parte de la "descripción" es correcta en términos muy generales. Sin embargo, no vemos en qué sentido ubica al interaccionismo simbólico como una perspectiva fenomenológica, algo que se señala sin aclaración alguna. Esta asignación sería parcialmente correcta para la etnometodología de Garfinkel (1967) que pone a trabajar elementos de la fenomenología social de Schutz para discutir a Parsons (Fornel, 2005). Así, un primer problema es la imprecisa generalidad con la cual Bourdieu construye esta suerte de tipo ideal llamado "modo de conocimiento fenomenológico". Sin embargo, desde su punto de vista, lo que emparienta a ambas teorías es compartir un primer "error", relativo a olvidar que:

...la verdad de la interacción no reside jamás enteramente en la interacción, cosa que olvidan la psicología social, el interaccionismo y la etnometodología cuando reduciendo la estructura objetiva de la relación entre los individuos agrupados, 
a la estructura coyuntural de su interacción en una situación y en un grupo particular intentan explicar todo lo que pasa en una interacción experimental u observada, por las características experimentales u observadas de la situación, como la posición relativa de los participantes en el espacio o los canales utilizados (Bourdieu, 1972, p.184).

La construcción de esta suerte de "conglomerado indigesto" -parafraseando libremente a Gramsci (1976)- donde bajo un mismo paraguas ubica perspectivas sociológicas con enormes diferencias entre sí -y al interior de cada una de ellas- sin un análisis en profundidad ${ }^{4}$, permite entender el rechazo que generan las críticas superficiales de Bourdieu a Schutz y/o Garfinkel, en aquellos que retoman la tradición fenomenológica en ciencias sociales (Throop y Murphy, 2002; Belvedere, 2011a, 2011b, 2012, 2013; Endress, 2005; Dreher, 2014, 2019) por estar basadas en lecturas parciales y reduccionistas. Particularmente en el caso de la etnometodología practicada por Garfinkel, se debe señalar que más que reducir las estructuras objetivas al orden interaccional este abordaje describe procedimientos mediante los cuales los agentes contribuyen a producir y reproducir prácticamente las "estructuras sociales" mediante prácticas de objetivación. Esto se ve con claridad ya en los trabajos pioneros agrupados por Garfinkel (1967, pp. 76-103) en Estudios en etnometodología, en especial en los capítulo 2 y 3, que tratan sobre el conocimiento de sentido común de las actividades y estructuras sociales, haciendo referencia a los escritos pioneros de Schutz (1993).

Sin embargo, desde la perspectiva de Perreau (2019), la primer crítica fuerte de Bourdieu al modo de conocimiento fenomenológico apunta a "la restricción del dominio de objeto de la fenomenología social” (Perreau, 2019, p. 74): ella "excluye la pregunta por las condiciones de posibilidad" de la relación primera, dóxica o "natural” con el mundo social (Bourdieu, 1972, pp.162-163; Bourdieu, 1980a, p. 44; Bourdieu, 1977). Eso la llevaría a no dar cuenta de las condiciones de clase desiguales que hacen a las diferentes relaciones dóxicas con el mundo social propias de diferentes habitus de clase y a las relaciones de dominación que se juegan en las interacciones.

La segunda crítica puede ser calificada de "metodológica” (Perreau, 2019, p. 75): las descripciones del orden interaccional al que se reduciría la estructura objetiva implica que Schutz y Garfinkel producen construcciones de segundo grado o informes de informes, como se señalará en Le sens pratique (Bourdieu, 1980a, pp. 44-45). Por el contrario, para Bourdieu (1972, pp. 184-185) lo que ocurre en la interacción (por ejemplo, "guardar o reducir las distancias") encuentra su verdad en la relación entre las posiciones de cada agente en las estructuras objetivas y las

4. Por ejemplo las diferencias entre Cicourel y Garfinkel (Miceli, 2006). 
disposiciones (habitus) que se constituyen en las trayectorias sociales que llevan a esa posición. En ambos casos, el modo de conocimiento fenomenológico no adoptaría una posición crítica (no vería la dominación) por quedar atrapado en una descripción del mundo dado como tal: esta sería la neutralización política de la fenomenología de la que hablará Bourdieu (1997, p. 249) en Méditations pascaliennes, tesis que ha sido objetada con justicia por Belvedere (2011b) y Dreher (2019).

\section{Las criticas de la critica}

Schutz suele ser señalado por Bourdieu como el representante de la "fenomenología social”. Sin embargo, como ha mostrado Belvedere (2011b, p. 41; 2012) contra el "disenso ortodoxo" de la teoría social contemporánea -encarnado por Bourdieu, Giddens y Habermas- su obra no encarna un "subjetivismo” sino que es un antecedente fundamental en la superación fenomenológica de la oposición dualista entre objetivismo y subjetivismo a partir de un "monismo complejo" (Belvedere, 201 1b, p. 86). Frente a la triple "reducción" ${ }^{5}$ de la obra de Schutz al subjetivismo, al constructivismo y al idealismo, impulsada por Bourdieu, Belvedere (2011b, pp. 41-94) muestra su inconsistencia -con un detalle que aquí no se puede reproducir- señalando que la noción de "mundo de la vida" (Lebenswelt) de Schutz (reelaborada en relación con la de Husserl) no se reduce "al mundo de la actitud natural" ni a un "conjunto de representaciones de sentido común articuladas a través del lenguaje” y "cuenta con una estructura social” (Belvedere, 2011 b, pp. 86, 88). Estas cuestiones fueron trabajadas en su obra póstuma, editada por Luckmann, cuyo título es significativo: Las estructuras del mundo de la vida (SLW en la cita siguiente) e indican lo incorrecta que es la crítica sobre la ausencia de una investigación sobre las condiciones de posibilidad de la relación con el mundo social como evidente (Bourdieu, 1980a, p. 44). Haciendo referencia a SLW, Belvedere señala que para Schutz:

El mundo de la vida está estratificado en diversos niveles y dimensiones, algunas de las cuales trascienden toda representación tales como la estructura espacial, la estructura temporal, el mundo físico, el tiempo objetivo, la historia y mi muerte (SLW, 166-167). Lo mismo vale para el mundo del sentido común; que trasciende la realidad de mi vida cotidiana en la medida en que presupone una forma social pre-organizada, la permanencia del mundo social más allá de mi muerte, la

\footnotetext{
5. Tal como lo aclara Belvedere (2011b) no se utiliza en este caso "reducción" en su "sentido técnico fenomenológico sino como operación restrictiva de la complejidad a representaciones unidimensionales y simplificadoras" (p. 48).
} 
organización de los hombres en grupos, la distancia social, y una secuencia de generaciones traslapadas (Belvedere, 2011b, p. 277).

Esto hace ver que Schutz, más que un adversario, indica Belvedere (2011a, 2012), bien podría haber sido considerado por Bourdieu como un antecedente a rescatar. En ocasiones esto ocurre, por ejemplo en la "entrevista sobre la fenomenología” concedida a Sapiro (2007) y en su Autoanálisis (Bourdieu, 2004).

Throop y Murphy (2002) -en un texto de referencia sobre este asuntorealizan una crítica de la crítica de Bourdieu a la fenomenología, indicando que se basa en una "mala interpretación” de Husserl y Schutz -como también dirán Endress (2005) y Dreher (2014, 2019) años después-. Afirman que las propuestas de Bourdieu presentan muchos puntos en común con Schutz y, como ejemplo, señalan que para el sociólogo austríaco los individuos "entran en un mundo social ya constituido que ha sido previamente creado por otras personas y comunidades" y que en la relación con ese mundo social, "con amigos, padres, maestros", adquieren un "conocimiento de primera mano que funciona como esquema de referencia en la comprensión de los aspectos particulares del entorno percibido" (Throop y Murphy, 2002, p. 196). Por el contrario,

Bourdieu ignora el hecho de que, a excepción de la noción de habitus como una cosa, todos los puntos que pretende encontrar en su relectura ya estaban presentes en los textos fenomenológicos (...) Bourdieu parece simplemente reformular algunas premisas de Schutz en su propio vocabulario idiosincrásico y excesivamente determinista para hacerlas sonar como nuevas, cuando en realidad no lo son (Throop and Murphy, 2002, p.197).

Si bien Throop y Murphy aciertan en el carácter sesgado de la crítica de Bourdieu a la fenomenología no es posible acordar con la imputación de "determinista”, lugar común de las críticas dirigidas contra él (Alexander, 1995). Aceptarla sería operar también una "mala interpretación" de su obra, tal como la que Bourdieu realiza sobre Schutz. Cabe señalar que Throop y Murphy construyen sus objeciones a partir de una lectura parcial de las investigaciones de Bourdieu, quedando fuera los análisis antropológicos sobre los ritos cabileños en los que presta particular atención al carácter indeterminado y ambiguo de la práctica (Bourdieu, 1980a, pp. 333-440), claramente inspirados en la perspectiva merleaupontyana (Dukuen, 2018b, pp. 183-233). Esto no exime a Bourdieu de realizar lecturas sesgadas de las teorías concurrentes, pero tampoco exime a sus 
críticos de obviar los análisis empíricos en los cuales se juegan las reapropiaciones teóricas.

Lo mismo se puede decir del carácter de reformulación que opera Bourdieu sobre nociones y problemáticas trabajadas por la fenomenología. En su "Response to Throop y Murphy”, escrita poco antes de morir (2002), señala que en la reasunción que realiza, las ideas teóricas provenientes de la fenomenología "están diseñadas para guiar la investigación empírica y para resolver problemas específicos de la antropología y la sociología... tales como el problema de intercambio de dones" (p. 209). Lejos de no tener novedad alguna, Bourdieu introduce el problema de la temporalidad y la formación de habitus corporales en el desarrollo de una teoría de los poderes y modos de dominación que no se encuentra en Husserl, Schutz o Merleau-Ponty, como se observa en los textos tempranos sobre la concepción del tiempo en los campesinos argelinos y en el abordaje de la hexis corporal de los célibes bearneses (Bourdieu, 1962, 1963; Dukuen, 2013, 2015; Dukuen, 2016, 2018b). Vale agregar que la relación entre hexis corporal, habitus y dominación es clave en obras posteriores como La distinction (Bourdieu, 1979), Le sens pratique (Bourdieu, 1980a) y Méditations pascaliennes (Bourdieu, 1997). En ese sentido, García (2012a), retomando análisis del libro La domination masculine de Bourdieu (1998), afirma:

Sus nociones de hexis y habitus resultan de una interpretación en clave política de la filosofía del cuerpo de Merleau-Ponty: brazos y piernas no solo tienen sus peculiares saberes, como dice Merleau-Ponty, sino que en la medida en que estos saberes se ajustan a normas culturales, brazos y piernas también "están colmados de imperativos mudos" (García, 2012a, pp. 378-370).

\section{El habitus en cuestión}

La noción de habitus ha sido objeto de numerosas críticas, entre las cuales ganó notoriedad la de Lahire (2004) quien, contra la estabilidad y unicidad que supone el concepto, señala el carácter plural de las disposiciones, achacando los defectos de la propuesta de Bourdieu a su fundamento en la fenomenología de Husserl y Merleau-Ponty ${ }^{7}$. Sin embargo, menos conocidas son las objeciones provenientes de la tradición fenomenológica que se analizarán en este apartado.

En una de sus paráfrasis a Husserl sobre la relación entre habitus, intencionalidad y temporalidad, Bourdieu (1987) señala:

7. Para una crítica de la posición de Lahire ver Frère (2011) y Dukuen (2018b). 
El habitus mantiene con el mundo social del que es producto una verdadera complicidad ontológica, principio de un conocimiento sin conciencia, de una intencionalidad sin intención, y de un dominio práctico de las regularidades del mundo que permite adelantar el porvenir sin tener ni siquiera necesidad de presentárselo como tal. Se encuentra allí el fundamento de la diferencia que hacía Husserl, en Ideas I, entre la protensión como un práctico tender hacia un porvenir inscripto en el presente, por lo tanto aprehendido como ya allí y dotado de la modalidad dóxica del presente, y el proyecto como posición de un futuro constituido como tal, es decir como pudiendo advenir o no advenir (p. 22).

Belvedere (2004) retoma esta cita para realizar una crítica centrada en la filiación entre el habitus y las nociones husserlianas de retensión y protensión: "más allá del carácter relativamente preciso de la referencia lo que no queda claro es de qué manera el habitus, en tanto incorporación de las estructuras sociales operada y operantes en las prácticas, podría fundar la temporalidad, puesto que más bien la presupone" (Belvedere, 2004, p. 67). Efectivamente, el habitus no puede ser el fundamento de la temporalidad, dado que se forma en y por la temporalidad que le presupone. En términos generales esto es correcto. Sin embargo, el planteamiento de Bourdieu también lo es, pero antes de explicar por qué, caben una serie de aclaraciones: el extracto citado es parte de una entrevista en la cual el autor se encuentra señalando sus diferencias con Sartre (1943) y Elster (1979), que desde otras posiciones filosóficas privilegiarían la conciencia reflexiva y el proyecto ${ }^{8}$. Como se señala en otros escritos (Dukuen, 2011, 2013, 2016, 2018b) en las investigaciones tempranas de Bourdieu (1963, 1964) sobre la sociedad cabileña se hace evidente la "antropologización” de los estudios de Husserl sobre la temporalidad (2002) que fueron su formación de base en filosofía y marco de su tesis doctoral inconclusa -dirigida por Canguilhem- sobre las "estructuras temporales de la vida afectiva” (Martínez, 2007; Dukuen 2013, 2018b).

Estas investigaciones antropológicas le permitieron a Bourdieu describir en acción la primacía práctica de una de las modalidades de la experiencia del tiempo que no es objetivante; es decir, la protensión, trabajada por Husserl: anticipación perceptiva fundada en la creencia y en la tradición (en un ethos), como inducción práctica de un porvenir cuasi presente. En la creencia o relación dóxica se asienta la "complicidad ontológica" (Bourdieu, 1980b, p. 7; 1987, p. 22) con el mundo, que implica una "intencionalidad sin intención” en el sentido de que no supone un proyecto objetivado por la conciencia objetivante sino operaciones corporales propias de la intencionalidad operante merleaupontyana. En un

8. Para una discusión de la crítica de Bourdieu a Sartre ver Hong (1999) y Dukuen (2018b). 
artículo previo (Bourdieu, 1980b) la referencia a la complicidad ontológica es remitida a Heidegger y Merleau-Ponty:

La relación con el mundo social no es la relación de causalidad mecánica que se suele suponer entre un "entorno" (milieu) y la conciencia, sino más bien una especie de complicidad ontológica: cuando la misma historia habita el hábito y el hábitat, las disposiciones y las posiciones, el rey y su corte, el empleador y su empresa, el obispo y su diócesis, la historia se comunica en cierto sentido consigo misma (...). La historia como "sujeto" se descubre en la historia como "objeto", ella se reconoce en las "síntesis pasivas", “antepredicativas”, estructuras estructuradas antes de toda operación estructurante y de toda expresión lingüística. La relación doxica con el mundo natal, esa suerte de compromiso ontológico que instaura el sentido práctico, es una relación de pertenencia y posesión en la cual el cuerpo, apropiado por la historia, se apropia de manera absoluta e inmediata de las cosas habitadas por la misma historia (10) (...) Esto es lo que me parece, que el último Heidegger y Merleau-Ponty (especialmente en Lo visible y lo invisible) trataron de expresar en el lenguaje de la ontología, es decir un más acá "salvaje” o "bárbaro" -yo diría simplemente práctico- de relación intencional con el objeto (Bourdieu, 1980b, pp. 6-7).

Hechas estas aclaraciones, Bourdieu no sugiere que el habitus es condición de posibilidad de la temporalidad en general sino que, en determinadas condiciones de existencia y dominios de la práctica (Dukuen, 2013, 2018b), los agentes en tanto cuerpos propios estructurados en habitus "privilegian” la modalidad protensional-retensional como relación con el mundo social, mientras que en otras condiciones de existencia y dominios de la práctica “privilegian” la rememoración secundaria y el proyecto. Para Bourdieu: a) la temporalidad preobjetiva es fundamento de la temporalidad objetiva, b) ambas modalidades son propias de la experiencia humana en general, siendo las diversas condiciones sociales encarnadas (por ejemplo las que diferencian al campesino cabileño, del obrero de los suburbios de Paris) las que fundamentan la primacía de una o de otra en tal o cual dominio de la práctica.

Cabe aclarar, además que el hecho de que Bourdieu ubique el habitus en el agente empírico y no en el sujeto trascendental, no implica desestimar ni "refutar” la perspectiva de Husserl (Bourdieu, 2002, p. 209), sino señalar niveles de análisis diferentes: en términos fenomenológicos podríamos decir que Bourdieu se ubicaría en la descripción de la actitud natural antes de la reducción trascendental, mientras que Husserl no renuncia a la subjetividad trascendental. Estas 
dos instancias son señaladas por Merleau-Ponty (1945, p. 419) $)^{9}$ y conducen a reflexiones sobre "el entrelazo empírico-trascendental” que exceden los límites de este trabajo (ampliar en Bourdieu, 1997; García, 2012; Dukuen, 2018b).

Estas aclaraciones permiten a continuación referir el problema de la fundamentación, señalado por Belvedere (2011a, p. 5): mientras que el habitus en Husserl es una posesión del ego, en Bourdieu no quedaría claro cuál es el substrato de la acción social, si el habitus o el agente. En la perspectiva husserliana "nunca es el habitus el que actúa o explica la acción social, sino que el ego concreto, dotado de un habitus permite entrar en una personalidad de un orden superior y convertirse en un agente social” (p. 5). Belvedere (2013) en un artículo posterior, propone una salida al problema de la fundamentación a partir de Schutz:

We can finally establish a hierarchy between the three overlapping elements in Bourdieu's work: social agents (or "social persons," as Schutz puts it) are founded on shared habitus, which are practical principles acquire by the personal ego in embodied actions in common environments or settings. Thus, it can be said that the phenomenolgical perspective grounds the agent in the habitus and the habitus in the monad. We now know that it is not the habitus itself which acts but the concrete embodied ego, endowed with a habitus which allows her/him to become in part a social person (Belvedere, 2013, p. 1106).

Al leer el trabajo de Belvedere de 2011, se había propuesto una fundamentación de orientación merleaupontyana del enfoque de Bourdieu centrada en una sociogénesis del habitus en el cuerpo propio, en el marco de la cual las prácticas son producto de un agente social que es un cuerpo parcialmente estructurado en términos de habitus. Esto supone, siguiendo a Merleau-Ponty, comprender al cuerpo-agente como un entrelazo entre dos dimensiones: cuerpo habitual y cuerpo actual (Dukuen, 2013). Posteriormente, tras leer el nuevo artículo de Belvedere (2013) se creyó necesario profundizar algunos puntos de esa sociogénesis (Dukuen, 2018b), los cuales se abordan en el apartado siguiente.

\footnotetext{
9. "Husserl en su última filosofía admite que toda reflexión debe empezar volviendo a la descripción del mundo vivido (Lebenswelt). Pero añade que para una segunda 'reducción' las estructuras del mundo vivido deben ser recolocadas a su vez en el flujo trascendental de una constitución universal en que todas las oscuridades del mundo se aclararían. Sin embargo, es manifiesto que hay que elegir entre dos cosas: o bien la constitución hace transparente el mundo, y no se ve por qué la reflexión tendría necesidad entonces de habérselas con el mundo vivido, o bien retiene algo de él y nunca despoja el mundo de su opacidad. El pensamiento de Husserl toma esta segunda dirección cada vez más, aunque con muchas reminiscencias del período logicista -como cuando convierte la racionalidad en un problema, cuando admite significaciones que, en último análisis son fluyentes (...) o cuando funda el conocimiento sobre una doxa originaria" (Merleau-Ponty, 1945, p. 419).
} 


\section{Para una reelaboración merleaupontyana del habitus en Bourdieu}

Comprender el habitus como una estructuración parcial del cuerpo propio, formado por diferentes capas disposicionales (primarias, secundarias, etc.) más o menos integradas, es decir, no necesariamente sistemáticas -Bourdieu y Sayad (1964) lo sugerían en Le déracinement- permite desarrollar teóricamente, por ejemplo, la posibilidad de transformación e incorporación de nuevas disposiciones más o menos contradictorias, cuestión que Bourdieu señala $(1982,1997)$ pero no fundamenta con claridad. También permite tensionar la crítica sobre el determinismo a la que hacían referencia Throop y Murphy, la cual se apoya en las tesis bourdeanas sobre el "ajuste" entre estructuras subjetivas (habitus) y objetivas -inspirado en Leibniz y Durkheim- y la histéresis del habitus como tendencia a perseverar en su ser-conatus en Spinoza- que explican la reproducción de las prácticas y del mundo social (Bourdieu, 1980a; Dukuen, 2013, 2018b, 2019).

Sin embargo -y esto es lo interesante- incluso en los mismos libros ${ }^{10}$ estas tesis entran en tensión con otras en las que Bourdieu insiste en que el habitus se instituye por una dialéctica (Bourdieu, 1972, pp. 163, 175, 178, 179; Bourdieu, 1980a, pp. 70, 88, 242) donde la lógica de la práctica y el sentido práctico son del orden de la ambigüedad y la indeterminación (Bourdieu, 1980a, pp. 28, 142, 146, 160, 177, 426-427), lo cual se ha analizado in extenso en Dukuen (2018b). Los supuestos de estas tesis provienen, especialmente, de la impronta fenomenológica de Husserl y Merleau-Ponty. Allí se ubican, por ejemplo, los análisis de Bourdieu (1980a, pp. 167-230) sobre el intercambio de dones y la violencia simbólica, donde el habitus es "sentido del juego" incorporado, disposiciones que permiten al agente desarrollar estrategias, es decir, apuestas de lucha (enjeux) frente a otros agentes, a partir de y por la apropiación/distribución desigual de los capitales específicos-eficientes (poderes) en aquellos dominios de la práctica y/o campos ${ }^{11}$ donde se encuentre envuelto.

Estas estrategias permiten jugar con el tempo, improvisar en situación y contribuyen a que el juego tenga su encanto, su imprevisibilidad, su incertidumbre (Bourdieu, 1980a, p. 168; Dukuen, 2018b). Bourdieu (1987) mismo señala que la noción de "estrategia", producida tanto contra la teoría del actor racional como

\footnotetext{
10. Como se ha mostrado in extenso mediante una lectura genética de la producción de los conceptos en Bourdieu (Dukuen 2018b), estas tensiones teóricas se ven con claridad en el desarrollo de una teoría de la práctica propiamente dicha, que va desde una primera cristalización en Esquisse (1972), pasando por Le sens pratique (1980) y La distinction (1979), hasta sus últimos elaboraciones en Méditations pascaliennes (1997).

11. Me refiero a "dominios de las prácticas" para dar cuenta de los "juegos sociales" estudiados por Bourdieu en sociedades "tradicionales" donde por definición no existen "campos" relativamente autónomos (i. e. el intercambio de dones en la sociedad cabileña) y para aquellos "juegos" que en las sociedades "modernas" se juegan "fuera de campo", como las relaciones afectivas. Sobre este punto, ver las discusiones relativas al alcance de la noción bourdeana de "campo" en Lahire (2004), Lemieux (2011) y Dukuen (2013, 2018b).
} 
contra el estructuralismo, es fruto de su lectura de Merleau-Ponty (Sapiro, 2007) y de una apropiación "libre" de los desarrollos de Wittgenstein sobre "seguir una regla” (Taylor, 1993).

En ese sentido, la propuesta de pensar el habitus como estructuración parcial del cuerpo propio (Dukuen, 2013) se inspira especialmente en la enseñanza de Merleau-Ponty y permite cuestionar las tesis objetivistas del "ajuste" y las histéresis, evitando la deriva en el determinismo y el mecanicismo, permaneciendo dentro de la praxeología bourdeana y de su fundamentación fenomenológica. Justamente por eso se sostiene aquí que más que el determinismo tout court que le atribuyen sus críticos, lo que se encuentra en Bourdieu es una tensión entre las tesis "objetivistas" y aquellas de raigambre fenomenológica (Dukuen, 2018b).

Para avanzar en este punto, hay que centrarse en la relación entre tres dimensiones del cuerpo en Merleau-Ponty, sobre la que se detiene García (2012b) en su exégesis: cuerpo orgánico, cuerpo habitual y cuerpo actual. Atendiendo a los análisis del fenomenólogo francés (Merleau-Ponty, 1945) sobre la anosognosia y el miembro fantasma, se observa que el mutilado no vive el cuerpo en términos de representación mental, ni con sus características observables en la actualidad, ni como suma de componentes anatomofisiológicos, sino como cuerpo habitual, como "repertorio de movimientos que han sedimentado por hábitos de comportamiento en el mundo". Se observa que entre el cuerpo actual "con sus características observables y que muevo a voluntad" y el cuerpo orgánico, se ubica el cuerpo habitual "que oficia de nexo y también de terreno ontológico común, del que ambos son dimensiones".

Es importante señalar que el fenómeno originario y central es el cuerpo habitual "como plataforma de despegue", y que las diferencias dimensiones se encuentran entrelazadas en la experiencia misma (García, 2012b, pp. 111, 112). Cabe indicar que la dialéctica entre cuerpo habitual y cuerpo actual es particularmente interesante porque se ubica en el dominio de investigación antropológica donde opera la praxeología.

A partir de estos señalamientos se propone repensar la noción de habitus en Bourdieu y comprenderla en el orden de las transformaciones prácticas: a) transformación de las "condiciones objetivas" a las experiencias prácticas; b) transformaciones en la incorporación de las experiencias prácticas como disposiciones; c) transformaciones en el pasaje de las disposiciones/esquemas a las prácticas (Dukuen, 2013, 2018b). Por cuestiones de espacio, no es posible detenerse en los puntos a y b-donde se tratan problemas relativos a la génesis de los diferentes modos de existencia y estratos del sentido- sino brevemente en el punto (c) transformaciones en el pasaje de las disposiciones a las prácticas.

Para ello, hay que tener en cuenta que en términos fenomenológicos cada puesta en juego de disposiciones por parte de un agente implica su transforma- 
ción en una práctica en situación en el mundo social práctico; una situación que solicita una acción, y que si bien puede tener diferentes grados de afinidad de sentido, nunca es la misma que formó la disposición. Entonces, si se considera que la práctica es resultado del encuentro entre un cuerpo-agente parcialmente estructurado por un habitus y una situación en un mundo social práctico que opera como solicitación, ese encuentro es centrífugo y centrípeto en términos de donación de sentido (Sinngebung) (Merleau-Ponty, 1945, p. 501).

Esta dialéctica implica que en la lógica de la práctica siempre hay un grado de indeterminación y ambigüedad, como ha señalado Merleau-Ponty (1942, 1945) y el propio Bourdieu en sus trabajos antropológicos sobre los cabileños (1980a, pp. 347-440) -ignorados por los críticos- y que el agente ante las solicitaciones del mundo puede inventar una respuesta a partir de sus disposiciones adquiridas. Ahora bien, el problema reside en qué poder de solicitación tienen las situaciones cuando no encuentran afinidad estructural de sentido con las disposiciones que el agente posee para responder. Esto depende del poder que se le otorgue al habitus en la estructuración del cuerpo propio.

En este punto puede ser de utilidad recordar el caso del "enfermo Schneider" estudiado por Merleau-Ponty (1945, pp.114-172). Schneider, quien había sufrido una lesión en la región occipital, estaba "encerrado" en su cuerpo habitual, en sus disposiciones adquiridas, y por lo tanto encontraba enormes dificultades para producir prácticas puestas en lo virtual, como jugar o realizar acciones "gratuitas” espontáneas. Solo podía realizar sus prácticas habituales, no así renovar su esquema corporal. En su caso, el entrelazamiento entre cuerpo habitual y cuerpo actual, sedimentación y trascendencia, se encontraba cortado. El caso le sirve a Merleau-Ponty para mostrar, por el contrario, que la dialéctica del cuerpo en el mundo se caracteriza por una "ambigüedad existencial" (Waldenfels, 1987, p.175; Ralón, 2005, p. 237): cuenta con la sedimentación de las disposiciones pasadas pero se encuentra abierta a lo posible. La temporalización se expresa en que en cada nuevo presente las disposiciones puedan resignificarse y, al mismo tiempo, se abre la posibilidad de crear otras.

En ese sentido, llevada al extremo, la tesis de Bourdieu sobre la histéresis del habitus primario formado en el microcosmos familiar, coarta la posibilidad de incorporar nuevas disposiciones salvo aquellas afines y tiende a transformar la dialéctica humana en una ficción retórica que limita al cuerpo a su dimensión habitual. Se evidencia que Bourdieu no distingue el doble aspecto del cuerpo: habitual y actual, ni toma en cuenta su entrelazamiento en la dialéctica con el mundo humano. De ahí la crítica que le realiza Bimbenet (2011) cuando señala que comparte con Merleau-Ponty la preocupación por lo arqueológico, pero se desentiende de lo teleológico. Por eso mismo, nuestra propuesta no solo debate 
con las perspectivas críticas fenomenológicas que hemos analizado, sino también con la propuesta de Bourdieu.

Se propone, entonces, recobrar el carácter dinámico del esquema corporal y postular que el cuerpo está parcialmente estructurado por un habitus en tanto cuerpo habitual y, por lo tanto, sigue siendo un "puedo" (Merleau-Ponty, 1945, p. 160), aunque no sea a cada paso un “yo”. Esto significa recuperar el carácter abierto del cuerpo actual en su entrelazamiento con el cuerpo habitual. Así, el habitus no estructura totalmente al agente, sino de manera parcial y justamente por eso podemos pensar la dialéctica del sentido práctico en su ambigüedad e indeterminación de manera positiva.

Ahora se está en condiciones de retomar el problema de la fundamentación señalado por Belvedere $(2011 \mathrm{~b}, 2013)$ y presentar un esbozo de sociogénesis del habitus: el cuerpo propio del niño/a se estructura a partir de las relaciones prácticas jerárquicas en el microcosmos familiar -o su equivalente práctico-donde se incorporan disposiciones/esquemas que conforman un habitus primario encarnado, principio de incorporación de las relaciones de poder y dominación relativas a la violencia simbólica de género, étnica y de clase ${ }^{12}$. En este nivel originario de incorporación/acción el que actúa es el cuerpo propio, y recuerda lo trabajado por Merleau-Ponty (1997) en su curso de la Sorbona sobre la formación del esquema corporal: "Las relaciones con el prójimo en el niño”.

Y en ese proceso de incorporación, en el cual se "forma" el cuerpo propio “infantil” (su esquema corporal) y se configura un habitus primario, va emergiendo lo que podemos llamar un cuerpo-agente social que produce prácticas en situación. En el marco de las diversas trayectorias sociales, cuando ese cuerpo-agente se introduce en otros dominios de la práctica (como la escuela) o campos, que implican otras situaciones-interacciones más o menos distantes, las disposiciones primarias son retrabajadas por nuevas prácticas y además se forman nuevas disposiciones secundarias a partir de la dimensión actual del cuerpo.

Entonces, acordamos con Belvedere en que en ningún caso es el habitus el que actúa, sino el cuerpo-agente social como entrelazo entre una dimensión parcialmente estructurada en términos de habitus y una dimensión actual, abierta a nuevas posibilidades. En la perspectiva que se desarrolla aquí a partir de Merleau-Ponty y Bourdieu, el cuerpo-agente social no está determinado ni cerrado por el habitus, sino condicionado por él, por las trayectorias sociales en las que se formó (con sus intersecciones de género, étnicas, de clase) y las relaciones prácticas específicas en las que se vea envuelto, que actúan como restricciones y posibilidades para adquirir nuevas disposiciones/esquemas, transformar antiguas

12. Bourdieu aborda estos temas desde un cruce entre sociología, antropología y psicoanálisis freudiano en Méditations pascaliennes y La domination masculine (Dukuen, 2018b, pp. 275-278). 
e incluso eliminarlas. La dimensión actual del cuerpo y la posibilidad de entrar en otras relaciones son las formas de apertura del cuerpo-agente en la dialéctica del mundo humano.

Esta propuesta implica debatir la crítica determinista a la teoría de la práctica mediante una ruptura con la "lógica del ajuste" entre habitus y condiciones objetivas, proponiendo además un enfoque que permite dar cuenta de las relaciones de poder y dominación de forma no mecanicista.

En ese sentido, comprender al cuerpo-agente como entrelazamiento habitual/actual da sentido a la noción de "contradiestramiento", posibilidad de transformación del habitus señalada por Bourdieu (1997, p. 248) en Méditations pascaliennes, la cual sería una ficción retórica si el cuerpo no tuviese una dimensión actual, abierta a lo posible. También esto le daría sentido a la propuesta de una "antropología reflexiva" (Bourdieu, 1980a; Bourdieu y Wacquant, 1992), en la medida en que para poder "objetivar la objetivación” de manera efectiva, y más generalmente, para que la toma de distancia crítico-reflexiva con las condiciones de existencia incorporadas produzca transformaciones duraderas en los cuerpos y prácticas, el cuerpo tiene que tener un poder instituyente de sentido (dimensión actual), que le permita aprehender prácticas reflexivas metadiscursivas e incorporarlas como disposiciones/esquemas corporales.

Ahora bien, la dimensión abierta y trascendente del cuerpo, de raigambre merleaupontyana es un aporte sustancial para la antropología crítica de la dominación propuesta por Bourdieu. Analizar los modos de dominación, describir cómo se somatizan, tiene sentido en su perspectiva en la medida en que los dominados puedan aprehender disposiciones críticas reapropiándose de sus experiencias, o sea que el cuerpo puede trabajarse mediante un contradiestramiento que solo es posible si el habitus no es una totalidad cerrada que se autodespliega. Esto significa efectivamente que el cuerpo habitual configura una "libertad condicionada", aquella que Merleau-Ponty (1945, p. 518) retomaba de Husserl como el suelo de nuestra existencia: allí se depositan también disposiciones relativas a las relaciones de dominación. Pero si es cierto que hay "una libertad condicionada y condicional" y "un margen de libertad" como dice Bourdieu (1980a, p. 92, 1997, pp. 236-240) es necesario renunciar a los extremos puros del determinismo y la libertad, para reconocer la existencia de grados de dominación (Nordmann, 2010).

En efecto, el cuerpo guarda un poder de institución de sentido, una dimensión abierta a lo virtual (cuerpo actual), que puede permitir que en relaciones prácticas duraderas con los otros en trayectorias sociales específicas y en experiencias colectivas, se incorporen disposiciones "rebeldes" que mediante un contradiestramiento reelaboren disposiciones dominadas, en "los márgenes de maniobra dejados a la libertad, es decir, a la acción política” (Bourdieu, 1993, p. 944). 
Se puede ilustrar este punto a partir de un caso práctico. En el marco de un trabajo de campo reciente sobre jóvenes, escuela, moral y política ${ }^{13}$, se analizaron las trayectorias de dos becarias de diecisiete años de clase media baja escolarizadas en un colegio secundario privado bilingüe para clases altas de Ciudad de Buenos Aires (Kriger y Dukuen, 2017; Dukuen, 2018a). En el marco de trayectorias de clase atípicas que rompen con la "causalidad de lo probable" (Bourdieu, 1979), en su día a día ellas transitan socializaciones no lineales donde circulan agentes y experiencias prácticas de clase social profundamente heterogéneas: se observa una marcada discontinuidad social y cultural cuando pasan de sus hogares y barrios al colegio.

En esas experiencias se pudo captar procesos de contradiestramiento donde se reelaboraron ciertas disposiciones, se incorporaron nuevas y otras desaparecieron, instituyendo habitus escindidos (Bourdieu, 1993; 1997) que componen diversos grados de complicidad ontológica y de tensión con esos mundos sociales. Esa heterogeneidad y escisión de las experiencias y disposiciones de las becarias se expresan en su lucidez crítica sobre lo político. Ellas se declaran "de izquierda" a partir de experiencias en agrupaciones políticas: una excepción tanto frente a sus compañeros de clases altas y al mundo "otro" que las rodea en la escuela, al cual examinan minuciosamente, como frente a sus propias familias; reconociendo al mismo tiempo la oportunidad única de una educación de elite.

Este caso práctico no es comprensible a partir de una lógica que se mueve entre los extremos puros del ajuste o del desajuste con las condiciones objetivas, o de la histéresis del habitus, que hubiese impedido el contradiestramiento. Por eso, vislumbrar estas posibilidades implica salir de la "mala dialéctica", criticada por Merleau-Ponty (1964, pp. 127-128), y comprender los condicionamientos sociales, las relaciones de poder y de dominación en el marco de la dialéctica ambigua de las prácticas sociales.

\section{Conclusiones}

En este trabajo se analizaron las críticas de Bourdieu a la "fenomenología social”, así como las objeciones de Throop y Murphy (2002) y Belvedere (2011a, 2011 b) quienes aciertan al indicar el carácter equívoco de su lectura de Schutz. Acordando en este punto, en el caso de Throop y Murphy se rechaza la acusación sobre el determinismo y la falta de aportes "novedosos" de Bourdieu.

13. Investigación dirigida por la Dra. Kriger (PICT 2012-2751 - 2014-2017). Entre 2015-2016 se realizaron entrevistas semi-estructuradas en el colegio a 15 estudiantes de 5to año. Entre 2016-2018 el Dr. Dukuen realizó trabajo de campo, "observación participante" en diferentes actividades escolares y más de 50 entrevistas con estudiantes, docentes, preceptores y directivos (Dukuen, 2018a). 
Yendo aún más allá de lo que Bourdieu (2002) señala en su respuesta a Throop y Murphy, se afirma que introduce el problema de la temporalidad y la formación de habitus corporales en el desarrollo de una teoría de la dominación que no encontramos en Husserl, Schutz o Merleau-Ponty, como se observa desde sus primeros trabajos antropológicos sobre los campesinos argelinos y los célibes bearneses (Bourdieu, 1962, 1963, 1980a). Así, este debate se enfrenta a una paradoja: quienes critican las lecturas simplistas de Bourdieu sobre la fenomenología, hacen exactamente lo mismo al no darle entidad a sus investigaciones empíricas, haciendo gala de teoricismo.

Luego, tras revisar las críticas de Belvedere a la noción de habitus y el problema de la fundamentación de la temporalidad y la acción, se ha propuesto una reelaboración basada en Merleau-Ponty, sin abandonar la praxeología bourdeana. Consiste en comprender al cuerpo-agente como parcialmente estructurado por un habitus que se encuentra entrelazado a una dimensión actual, abierta a lo posible. Ciertamente, esta propuesta se realiza también con y contra Bourdieu, en la medida en que pugna contra la tensión entre las tesis sobre el ajuste-histéresis del habitus (que fundamentan las acusaciones de determinismo) y las tesis provenientes de la impronta fenomenológica de la praxeología. Por eso mismo, recuperando la dialéctica entre cuerpo habitual y cuerpo actual, no desarrollada por Bourdieu, se puede fundamentar con mayor solidez una antropología reflexiva, como vocación liberadora clave de la teoría de los poderes y modos de dominación. Contra el teoricismo, la breve referencia a la investigación sobre de las becarias pretendió ser una ilustración empírica de este enfoque.

En un escrito elocuente, el especialista en Merleau-Ponty, Étienne Bimbenet señaló que "la definición bourdiana del sentido práctico, y correlativamente del habitus, pueden ser leídas como una ejemplificación sociológica fiel de la fenomenología merleaupontyana de la percepción" (2011, p. 167). Con este trabajo se espera haber contribuido a restituir la praxeología de Bourdieu a las ciencias sociales de orientación fenomenológica.

\section{Agradecimientos}

El autor agradece los comentarios de los evaluadores que ayudaron a clarificar el argumento de este trabajo.

\section{Referencias}

Alexander, J. (1995). Fin de Siècle Social Theory. Londres, Inglaterra: Verso. 
Aristóteles (1997). Éthique a Nicomaque. París, France: Vrin.

Belvedere, C. (2004). Intención e intencionalidad en las críticas de la teoría social a Schutz. En E. Ipola (Coord.). El eterno retorno. Acción y sistema en la teoría social contemporánea (pp. 57-78). Buenos Aires, Argentina: Biblos.

Belvedere, C. (2011a). Bourdieu's Concept of Habitus as a Substruction of the Monad. Filadelfia, EEUU: Society for Phenomenology and the Human Sciences.

Belvedere, C. (2011b). Problemas de fenomenología social. A propósito de Alfred Schutz, las ciencias sociales y las cosas mismas. Buenos Aires, Argentina: UNGS-Prometeo.

Belvedere, C. (2012). El discurso del dualismo en la teoría social contemporánea. Una crítica fenomenológica. Buenos Aires, Argentina: Eudeba.

Belvedere, C. (2013). The Habitus Made Me Do It: Bourdieu's Key Concept as a Substruction of the Monad. Philosophy Study, 3(12), 1094-1 108. https://ri. conicet.gov.ar/handle/11336/3714 [Consultado el 13 de junio de 2019].

Bimbenet, É. (2011). Sens pratique et pratiques réflexives. Après Merleau-Ponty: études sur la fécondité d'une pensée. París, Francia: Vrin.

Bourdieu, P.; Sayad, A. (1964). Le déracinement. París, Francia: Minuit.

Bourdieu, P.; Wacqüant, L. (1992). Réponses. Pour une anthropologie réfléxive, París, Francia: Seuil.

Bourdieu, P. (1962). Célibat et condition paysanne. Études Rurales, 5(6), 32-136. https://www.persee.fr/doc/rural_oo 14-2182_1962_num_5_1_1011 [Consultado el 13 de junio de 2019].

Bourdieu, P. (1963). La société traditionnelle. Attitude à l'égard du temps et conduite économique. Sociologie du travail, 5, 24-44. https://www.persee.fr/ doc/sotra_0038-0296_1963_num_5_1_1127 [Consultado el 10 de julio de 2019].

Bourdieu, P. (1965). Introduction. Un art moyen. París, Francia: Minuit.

Bourdieu, P. (1972). Esquisse d'une théorie de la pratique. París, Francia: Droz. 
Bourdieu, P. (1977). Sur le pouvoir symbolique. Annales, 3, 405-411.

https://www.persee.fr/doc/ahess_0395-2649_1977_num_32_3_293828 [Consultado el 10 de febrero de 2019].

Bourdieu, P. (1979). La distinction. París, Francia: Minuit.

Bourdieu, P. (1980a). Le sens pratique. París, Francia: Minuit.

Bourdieu, P. (1980b). Le mort saisit le vif. Les relations entre l'historie réifiée et l'historie incorporée. Actes de la recherche en sciences sociales, 32(33), 3-14. https://dialnet.unirioja.es/servlet/articulo?codigo=5532374 [Consultado el 10 de julio de 2019].

Bourdieu, P. (1982). Ce que parleur veut dire? París, Francia: Fayard.

Bourdieu, P. (1987). Choses dites. París, Francia: Minuit

Bourdieu, P. (1993). La Misère du monde. París, Francia: Seuil.

Bourdieu, P. (1997). Méditations pascaliennes. París, Francia: Seuil

Bourdieu, P. (1998). La domination masculine. París, Francia: Seuil.

Bourdieu, P. (2002). Response to Throop \& Murphy. Anthropological Theory, 2(2), 209. https://journals.sagepub.com/doi/10.1177/14634996020020020701 [Consultado el 10 de febrero de 2019].

Bourdieu, P. (2004). Esquisse pour une auto-analyse. París. Francia: Raisons d'Agir.

Bourdieu, P. (2015). Sociologie générale 1. París, Francia: Raisons d’agir/Seuil.

Bourdieu, P. (2016). Sociologie générale 2. París, Francia: Raisons d’agir/Seuil.

Csordas, T. (2011). Modos somáticos de atención. En S. Citro (Coord.). Cuerpos plurales. (pp. 83-104).Buenos Aires, Argentina: Biblos.

De Aquino, T. (1993). Suma de Teología II. Madrid, España: BAC.

Dreher, J. (2014). Fenomenología del poder. En J. Dreher \& D. G. López (Comps.). Fenomenología del poder (pp. 25-50). Bogotá, Colombia: USTA. 
Dreher, J. (2019). The oblivion of power? In M. Pfadenhauer \& H. Knoblauch (Eds). Social Constructivism as Paradigm? (pp. 235-250). London, England: Routlegde.

Dukuen, J. (2010). Entre Schutz y Bourdieu. Encuentros y desencuentros en fenomenología social. RELACES 3, 39-50. http://www.relaces.com.ar/index. $\mathrm{php} /$ relaces/article/view/31/50 [Consultado el 11 de octubre de 2019].

Dukuen, J. (2011). Temporalidad, habitus y violencia simbólica. Génesis de una teoría de la dominación en la obra de Bourdieu. Avatares, 2, 152165. https://publicaciones.sociales.uba.ar/index.php/avatares/article/ view/4744/3875 [Consultado el 10 de febrero de 2019].

Dukuen, J. (2013). Habitus y dominación. Para una crítica de la teoría de la violencia simbólica en Bourdieu (Tesis doctoral, inédita). Universidad de Buenos Aires, Argentina.

Dukuen, J. (2015). Los usos del habitus en la génesis de las investigaciones antropológicas de Bourdieu. Prácticas de Oficio, 16, 1-25. https://ri.conicet. gov.ar/bitstream/handle/11336/17180/CONICET_Digital_Nro.20997. pdf? sequence $=1 \&$ isAllowed $=y$ [Consultado el 11 de octubre de 2019].

Dukuen, J. (2016). Ethos y temporalidad: una antropología de orientación fenomenológica en Bourdieu. Trabajo y Sociedad, 26, 141-153. https://www.redalyc. $\mathrm{org} / \mathrm{pdf} / 3873 / 387343599009$.pdf [Consultado el 10 de febrero de 2019].

Dukuen, J. (2018a). Socialización política juvenil en un colegio de clases altas (Buenos Aires, Argentina). Revista Latinoamericana de Ciencias Sociales, Niñez y Juventud, 16(2), 867-880. http://revistaumanizales.cinde.org. $\mathrm{co} / \mathrm{rlcsnj} /$ index.php/Revista-Latinoamericana/article/view/3508/957 [Consultado el 11 de octubre de 2019].

Dukuen, J. (2018b). Habitus y dominación en la antropología de Pierre Bourdieu. Una crítica desde la fenomenología de Maurice Merleau-Ponty. Buenos Aires, Argentina: Biblos.

Dukuen, J. (2019). Un arte de inventar. El habitus en la lectura bourdiana de Panofsky. Revista Cuadernos del Centro de Estudios en Diseño y Comunicación, $88, \quad 17-34 . \quad$ https://fido.palermo.edu/servicios_dyc/publicacionesdc/ cuadernos/detalle_articulo.php?id_libro $=784 \&$ id_articulo $=16261$ [Consultado el 10 de febrero de 2019]. 
Durkheim, E. (1990). L'evolution pédagogique en France. París: PUF.

Elster, J. (1979). Ulysses and the Sirens, Cambridge, UK, Cambridge UP.

Endress, M. (2005). Reflexivity, Reality, and Relationality. The Inadequacy of Bourdiue's Critique of the Phenomenological Tradition in Sociology. En M. Endress, G. Psathas, H. Nasu (Eds.). Explorations of the Life-world (pp. 51-74). Dordrecht, Holanda: Springer.

Fornel, M. (2005). Habitus y etnométodos. En P. Encrevé y R. S. Lagrave (Eds.). Trabajar con Bourdieu (pp. 25-45). Buenos Aires, Argentina: Ed. Flammarion.

Frére, B. (2011). Bourdieu's Sociological Fiction: A Phenomenological Reading of Habitus. En S. Susen \& B. S. Turner (Eds.). The Legacy of Pierre Bourdieu (pp. 247-270). Londres, Inglaterra: Anthem Press.

García, E. (2012a). Merleau-Ponty en la reflexión contemporánea acerca de las políticas normativas del cuerpo. En M. T. Ramírez (Coord.). Merleau-Ponty viviente (pp. 345-370). México, Siglo XXI.

García, E. (2012b). Maurice Merleau-Ponty. Filosofía, corporalidad y percepción. Buenos Aires, Argentina: Rhesis.

Garfinkel, H. (1967). Studies in Ethnometodology. New Jersey, EE UU: Prentice Hall.

Gramsci, A. (1976). Cuadernos de la Cárcel. México, D. F.: Juan Pablo Editor.

Haber, S. (2004). La sociologique française contemporaine devant le concept bourdieusien d'habitus. Alter. Revue de phénoménologie, 12, 191- 215.

Heran, F. (1987). La seconde nature de l'habitus. Tradition philosophique et sens commun dans le langage sociologique". Revue française de sociologie, 28(3), 385-416 https://www.persee.fr/doc/rfsoc_0035-2969_1987_ num_28_3_2423 [Consultado el 10 de febrero de 2019].

Hong, S. M. (1999). Habitus, corps, domination. Sur certains présupposés philosophiques de la sociologie de Pierre Bourdieu. París, Francia: L'Harmattan.

Husserl, E. (1966). Méditations cartésiennes. París, France: Vrin. 
Husserl, E. (2002). Lecciones sobre la fenomenología de la conciencia interna del tiempo. Madrid, España: Trotta.

Kriger, M.; Dukuen, J. (2017). Haciendo de la necesidad virtud: Socialización política y herencia familiar entre becarias de un colegio de clases altas. Pilquen, 20(3), 67-81. https://dialnet.unirioja.es/servlet/articulo?codigo=6128189 [Consultado el 11 de octubre de 2019].

Lahire, B. (2004). El hombre plural. Barcelona, España: Belaterra.

Lemieux, C. (2011). Le crépuscule des champs. En M. Fornel et al. (Eds.). Bourdieu, théoricien de la pratique (pp. 75-100). París, Francia: Ed. EHESS.

Lévi-Strauss, C. (2001). Antropología estructural: mito, sociedad, humanidades. México, D. F.: Siglo Veintiuno.

Martin-Criado, E. (2006). Estudio introductorio: las dos Argelias de Pierre Bourdieu. En P. Bourdieu. Sociología de Argelia y Tres estudios de etnología cabilia (pp. 15-119) Madrid, España: CEI-BOE.

Martínez, A. T. (2007). Pierre Bourdieu. Razones y lecciones de una práctica sociológica. Buenos Aires, Argentina: Manantial.

Mauss, M. (1979). Técnicas y movimientos corporales. En M. Mauss. Sociología y Antropología (pp. 337-356). Madrid, España: Tecnos.

Merleau-Ponty, M. (1942). La structure du comportament. París, Francia: PUF.

Merleau-Ponty, M. (1945). Phénomènologie de la perception. París, Francia: Gallimard.

Merleau-Ponty, M. (1964). Le visible et le invisible. París, Francia: Gallimard.

Merleau-Ponty, M. (1997). Les relations avec autrui chez l'enfant. Parcours, 19351951. París, Francia: Verdier.

Miceli, S. (2006). Entrevista com Aaron V. Cicourel. Tempo Social, revista de sociologia da USP, 19(1), 131-168. http://www.scielo.br/pdf/ts/v19n1/ a08v19n1 [Consultado el 11 de octubre de 2019]

Nordmann, C. (2010). Bourdieu/Rancière. Buenos Aires, Argentina: Nueva Visión. 
Perreau, L. (2019). Bourdieu et la phénoménologie. París, Francia: CNRS.

Ralón, G. (2005). La intencionalidad en el interior del ser: las homologías entre los sistemas simbólicos (Tesis doctoral, inédita). Universidad de Buenos Aires, Argentina.

Ralón, G. (2010). La lógica práctica y la noción de hábito. Anuario Colombiano de Fenomenología, IV. 243-261. https://www.unicauca.edu.co/fchs/sites/ default/files/Anuario\%20Colombiano\%20de\%20Fenomenolog\%C3\%ADa\%20.pdf [Consultado el 11 de octubre de 2019].

Ralón, G.; Dukuen J. (2013). Los modos de dominación en la socio-antropología de Bourdieu. Esbozo de una crítica. Estudios de Filosofía, 47, 9-33. http://www.scielo.org.co/pdf/ef/n47/n47a01.pdf [Consultado el 11 de octubre de 2019].

Sapiro, G. (2007). P. Bourdieu sobre la fenomenología. En P. Champagne et al. (Eds.). Pierre Bourdieu, sociólogo (pp. 59-66). Buenos Aires, Argentina: Nueva Visión.

Sartre, J. P. (1943). L'être et le néant, París, Francia: Gallimard.

Saussure, F. (1980). Curso de lingüística general. Buenos Aires, Argentina: Losada S. A.

Schutz, A. (1993). La construcción significativa del mundo social. Barcelona, España: Paidós.

Schutz, A.; Luckmann, T. (2003). Las estructuras del mundo de la vida. Buenos Aires, Argentina: Amorrortu.

Taylor, Ch. (1993). To Follow a Rule. C. Calhoun, E. LiPuma, M. Postone (Eds.). Bourdieu: Critical Perspectives (pp. 45-60). Chicago, EE UU: The University of Chicago Press.

Throop, J.; Murphy, K. (2002). Bourdieu and Phenomenology: A Critical Assessment. Anthropological Theory, 2(2), 185-207.

https://journals.sagepub.com/doi/10.1177/1469962002002002630 [Consultado el 11 de octubre de 2019].

Weber, M. (2009). La ética protestante y el espíritu del capitalismo. Buenos Aires, Argentina: Caronte. 